\title{
Technique of Hydrothermal Power System Balance Calculation with Allowance for the Regime Features of a Hydropower Station Based on the Cost of a Hydraulic Resource
}

\author{
Yuri A. Sekretarev and Tatiana V. Myatezh* \\ Novosibirsk State Technical University \\ Novosibirsk, Russian Federation
}

Received 20.07.2021, received in revised form 10.08.2021, accepted 04.09.2021

\begin{abstract}
The present paper proposes a universal method for analyzing the efficiency of technical systems using the combination of an optimization method and a method of marginal utility evaluation. At present, the problem of optimal load distribution in the power system between a hydropower plant (HPP) and thermal power plants (TPP) is solved using the equality of the differential incremental rate characteristics of fuel consumption at TPP and water consumption at HPP by the Lagrangian multiplier method. In this case, the number of iterations can be five or more. The proposed approach is based, first of all, on the correct representation of the differential characteristics and calculation of a hydro resource price for the operational control of the HPP. Based on the comparison of water volume at a HPP and fuel amount at combined heat and power plants (CHPP) used for generation of $1 \mathrm{~kW}$ power, it is possible to determine a water price for a HPP. Using the examples of Novosibirsk HPP and CHPP, it is expected to develop an estimation of economic effect from the implementation of the developed criteria, the proposed method of determination of a water price at HPP. As a result of implementing the developed method for the HPP, a price of sold electricity in the flexible energy market will be comparable with the price for sold electricity produced at CHPPs, being equal to approximately 12 rubles $/ \mathrm{kW} \cdot \mathrm{h}$.
\end{abstract}

Keywords: operational control of hydropower plants, optimization, hydro resource price for hydropower plants, incremental water rate characteristic, electricity market, complex criteria of ecological-andeconomic efficiency.

Citation: Sekretarev Yu.A., Myatezh T. V. Technique of hydrothermal power system balance calculation with allowance for the regime features of a hydropower station based on the cost of a hydraulic resource, J. Sib. Fed. Univ. Eng. \& Technol., 2021, 14(6), 641-655. DOI: 10.17516/1999-494X-0340

(C) Siberian Federal University. All rights reserved

This work is licensed under a Creative Commons Attribution-Non Commercial 4.0 International License (CC BY-NC 4.0).

* Corresponding author E-mail address: tatianamyateg@mail.ru 


\title{
Методика построения баланса в гидротепловой энергосистеме с учетом режимных особенностей ГЭС на основе определения стоимости гидроресурса
}

\author{
Ю. А. Секретарев, Т. В. Мятеж \\ Новосибирский государственный технический университет \\ Российская Федерация, Новосибирск
}

\begin{abstract}
Аннотация. Разработан универсальный метод, представляющий собой сочетание оптимизационного метода и способа оценки предельной полезности. На его основе целесообразно решать задачу краткосрочной оперативной оптимизации распределения нагрузки в гидротепловых электроэнергетических системах. В настоящее время задача оптимального распределения нагрузки энергосистемы между ГЭС и ТЭС решается на основе равенства дифференциальных характеристик расхода топлива на тепловых электростанциях и расхода воды на гидравлических с использованием метода неопределенных множителей Лагранжа. При этом число итераций может составлять пять и более. Предлагаемый подход основывается, прежде всего, на корректном представлении дифференциальных характеристик и определении стоимости гидроресурса для оперативного управления режимами работы ГЭС. На основе сопоставления объема воды, используемой на ГЭС, и количества топлива на ТЭЦ, затрачиваемого для выработки 1 кВт мощности, появляется возможность определить цену воды для ГЭС. На примере новосибирских ГЭС и ТЭЦ ожидается получение оценки экономического эффекта от внедрения разработанных критериев, предложенной методики по определению цены воды для ГЭС, а также методики разнесения топливных затрат на ТЭЦ. По результатам реализации разрабатываемого подхода на ГЭС цена продаж электроэнергии на гибком энергетическом рынке будет сопоставима с ценой продаж электроэнергии, вырабатываемой на ТЭЦ, и составит примерно 12 коп/кВт*ч.
\end{abstract}

Ключевые слова: оперативное управление режимами работы ГЭС, стоимость гидроресурса для ГЭС, характеристика относительных приростов расхода воды, рынок электроэнергии.

Цитирование: Секретарев, Ю.А. Методика построения баланса в гидротепловой энергосистеме с учетом режимных особенностей ГЭС на основе определения стоимости гидроресурса / Ю. А. Секретарев, Т. В. Мятеж // Журн. Сиб. федер. ун-та. Техника и технологии, 2021, 14(6). С. 641-655. DOI: 10.17516/1999-494X-0340

\section{Введение}

В статье представлена математическая модель определения стоимости гидроресурса для ГЭС при оптимизации режимов работы станций, разработанная на основе комплексных критериев эколого-экономической эффективности $[1,2]$. Сутью модели является сочетание оптимизационного метода неопределенных множителей Лагранжа с теорией предельной полезности, позволяющей на основе сопоставления объема воды, используемой на ГЭС, и количества топлива на ТЭЦ, затрачиваемого для выработки 1 кВт мощности, определить цену воды для ГЭС $[3,4]$. Полученный результат дает возможность эффективно и просто составлять оптимальный баланс мощностей в энергосистеме.

\section{1. Математическая модель}

\section{определения стоимости гидроресурса на ГЭС}

Водные ресурсы играют важную роль в экономии первичных энергетических и материальных ресурсов на входе в техническую систему, а в конечном итоге в снижении вредного 
воздействия на человека и окружающую природную среду $[5,6]$. Общим правилом является следующее: выгоднее экономить энергетические и материальные ресурсы в процессе реализации технологических процессов, как можно ближе к конечным участкам, т.е 1 кВт•ч энергии в рублях стоит в конце технологической цепочки намного больше, чем в начале [7].

Для определения стоимости гидроресурса на ГЭС для ее оперативного управления используем теорию предельной полезности.

Предельная полезность - это увеличение общей полезности при потреблении одной дополнительной единицы блага (производная):

$$
M U=\frac{\partial U}{\partial Q}
$$

где $M U$ - функция полезности; $U$ - функция полезности; $Q$ - количество потребляемого блага.

Принцип предельной полезности сводится к следующему: ценность блага данного рода определяется полезностью предельного экземпляра, удовлетворяющего наименее настоятельную потребность. Предельная полезность определяет спрос на товар [8].

В качестве продуктов будем рассматривать воду и топливо. А производную от них (т. е. относительный прирост расхода топлива на ТЭЦ (b) и воды (q) на ГЭС) используем как способ определения предельной полезности, рассчитанной по выражению (1).

Соображения относительно предельной полезности можно представить в очень простой графический форме с использованием линий безразличия (рис. 1). При этом согласно правилам построения необходимо взять величины, обратные от b и q, т. е. $1 / \mathrm{b}$ и $1 / \mathrm{q}$, и отложить соответственно по осям абсцисс и ординат, после чего соединить эти точки линией, которая и будет называться линией безразличия (рис. 1).

Обратные величины берут, чтобы показать полезность от использования продуктов. Так, на нашем примере получаем 1/b $\left[\mathrm{MBT}^{*} \mathrm{q} /\right.$ т. у. т.] и 1/q $\left[\mathrm{MBT}^{*}{ }^{4} / \mathrm{M}^{3}\right]$ соответственно, т. е. полезность для потребителя электроэнергии от использования 1 т. у. т. топлива и 1 м $^{3}$ воды соответственно [8].

\section{2. Математическая модель управления режимами работы смешанной энергосистемы}

В настоящей работе изложен универсальный метод, представляющий собой сочетание оптимизационного метода и способа оценки предельной полезности [9]. На его основе целе-

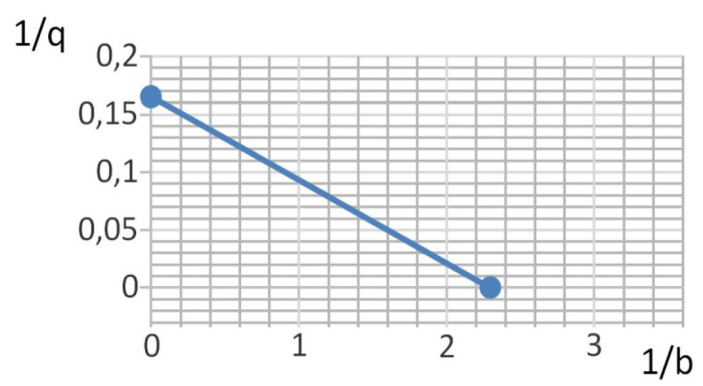

Рис. 1. Линия безразличия для периода естественной приточности (напор 17,9 м)

Fig. 1. Indifference line for the natural afflux period (the head of $17.9 \mathrm{~m}$ )

$$
-643-
$$


сообразно решать задачу краткосрочной оперативной оптимизации распределения нагрузки в гидротепловых электроэнергетических системах.

Рассмотрим более подробно, в чем заключается новизна и эффективность предлагаемого метода по сравнению с существующим подходом.

В настоящее время задача оптимального распределения нагрузки энергосистемы между ГЭС и ТЭС решается на основе равенства дифференциальных характеристик расхода топлива на тепловых электростанциях и расхода воды на гидравлических с использованием метода неопределенных множителей Лагранжа. Такие характеристики еще в СССР получили названия характеристик относительных приростов (ХОП) [10].

В общем виде критерий оптимального распределения нагрузки в ЭЭС без учета технических ограничений, накладываемых на него, выглядит следующим образом:

$$
b_{1}=b_{2}=\ldots=b_{n}=\lambda q=i d e m .
$$

Здесь $b_{1}, b_{2}, \ldots, b_{n}$ - относительные приросты расходов топлива на тепловых станциях (ТЭС), число которых в ЭЭС в общем случае равно $n$;

$q$ - относительный прирост расхода воды на гидростанции (ГЭС);

$\lambda$ - переводной коэффициент, суть которого будет раскрыта ниже.

Следует заметить, что ТЭС в оптимизационных задачах представляются как генерирующие источники с «неограниченным энергоресурсом» [8]. Это подразумевает, что в отдельно взятый момент времени любая мощность станции, естественно, в пределах допустимого диапазона ее работы будет обеспечена запасом энергетического ресурса вне зависимости от того, какую мощность станция несла в предшествующий момент времени. Это дает основание объединять все тепловые станции в одну эквивалентную с обязательным учетом всех технических ограничений [9].

Гидравлические станции относятся к генерирующим источникам с «ограниченными энергоресурсами», так как их количество определяется гидрографом реки и конечной полезной емкостью водохранилища [10]. Это говорит о том, что мощность ГЭС в данный момент времени зависит от того, с какой мощностью станция работала в предыдущий временной интервал. Поэтому гидравлические станции эквивалентировать невозможно, так как каждая из них уникальна с учетом вышеперечисленных условий.

Дифференциальные расходные характеристики ГЭС и ТЭС имеют различную размерность, а именно:

$$
\begin{aligned}
& b=\frac{d B}{d N}, \\
& q=\frac{d Q}{d N},
\end{aligned}
$$

где $B$ - расход топлива (тут/час), а $Q$ - расход воды (м³/c).

Поэтому коэффициент $\lambda$ в выражении (2) выполняет роль переводного множителя и называется мерой эффективного использования гидроресурсов в ЭЭС [11]. Поэтому приходится опытным путем подбирать значение коэффициента $\lambda$ с учетом также и ограниченности гидроресурса на ГЭС.

При этом число итераций может составлять от пяти и более до тех пор, пока не будет выполнено условие (2). Эти обстоятельства приводят к серьезному усложнению расчетов, свя- 
занных, прежде всего, с увеличением количества итерационных процедур, а значит, и времени решения и сходимостью данного процесса [12].

Предполагается, что на гидростанции в течение периода оптимизации напор не меняется, хотя станция и ведет регулирование. Такие случаи встречаются для высоконапорных и средненапорных ГЭС, когда изменение напоров за счет колебания бьефов не вносит существенной погрешности в энергетические показатели станции. Как будет видно дальше, допущение о постоянстве напора ГЭС существенно упрощает алгоритм решения задачи [13]. При этом $1 \mathrm{~m}^{3}$ воды для всего периода оптимизации обладает практически одинаковой энергией.

При распределении суммарной нагрузки в системе, состоящей из ТЭС и ГЭС, необходимо знать характеристики относительных приростов расхода условного топлива. Влияние гидростанции на это распределение учитывается с помощью коэффициента энергетической эффективности воды $\lambda$ для ГЭС, который устанавливает взаимосвязь между относительными приростами расхода условного топлива на ТЭС и относительными приростами расхода воды на ГЭС. Произведение коэффициента энергетической эффективности ГЭС $(\lambda)$ на ее относительный прирост расхода топлива представляет собой приведенный относительный прирост гидроэлектростанции по условному топливу [14].

Таким образом, условием экономичности совместной работы ТЭС и ГЭС в системе является то, что они должны в каждый момент времени работать с нагрузками, соответствующими одинаковым значениям относительных приростов расхода топлива на ТЭС и приведенными к условному относительных приростов на ГЭС. При решении задачи оптимизации смешанной энергосистемы необходимо учесть ограничения по заданному расходу воды из водохранилища на ГЭС. Для этого выполняется водно-энергетический расчет (ВЭР), а также необходимо подобрать оптимальное значение коэффициента энергетической эффективности $\lambda$. Значение коэффициента $\lambda$ определяется подбором, для данной ГЭС принимается постоянным в течение суток, зависящим от заданного суточного расхода воды.

Составим математическую модель и алгоритм для реализации поставленной цели.

\section{Математическая модель}

1. Уравнение цели

$$
\begin{aligned}
U & =\sum_{t} P_{T Э C t}+U_{T Э C t} \Rightarrow \min \\
U & =\sum_{t} P_{\Gamma^{\ni} Э t}+U_{\Gamma Э C t} \Rightarrow \min \\
U & =U+U^{\prime} \Rightarrow \min
\end{aligned}
$$

2. Уравнение связи

$$
\begin{aligned}
& B_{\text {ТЭС }}\left(P_{\text {ТЭС }}\right) \text { при } Q_{\text {ТЭС min }} \leq Q_{\text {ТЭС }} \leq Q_{\text {TЭС max }} \\
& Q_{\Gamma \ni C}\left(P_{Г \supset С}\right) \text { при } Q_{\Gamma \ni С \text { min }} \leq Q_{\Gamma \supset C} \leq Q_{\Gamma \supset С \max } .
\end{aligned}
$$

3. Уравнение ограничений

$$
\begin{aligned}
& P_{T Э C \text { min }} \leq P_{T Э C} \leq P_{\text {TЭС max }}, \\
& P_{\Gamma \ni C \text { min }} \leq P_{\Gamma \ni C} \leq P_{\Gamma \ni C \text { max }},
\end{aligned}
$$




$$
\begin{aligned}
& P_{\Gamma \ni C}+P_{T Э С}-P_{H}=0 \\
& \bar{Q}_{c p . c y m . Г \ni С}=\bar{Q}_{c p . з а д . Г \ni С} .
\end{aligned}
$$

4. Уравнение оптимизации

$$
\begin{aligned}
& U_{T \ni C t}=i d e m \\
& U_{\Gamma \ni C t}=i d e m,(\lambda * q=b),
\end{aligned}
$$

где $b$ и $q$ - характеристики относительных приростов расхода топлива и воды на эквивалентной ТЭС и ГЭС соответственно; $\mathrm{P}_{\mathrm{C}}, N_{\text {ТЭС }}, N_{\text {Гэс }}-$ нагрузка энергосистемы, мощности покрытия нагрузки эквивалентной ТЭС и ГЭС соответственно; $N_{\text {ТЭс } \min }, N_{\text {TЭС } \max }, N_{\text {Гэс min }}$, $N_{\text {гэс max }}$ - минимальная и максимальная мощности эквивалентной тепловой и гидравлической станций соответственно: $Q_{3 \text { дд }}$ предельно допустимая величина расхода воды на ГЭС, определяемая водно-энергетическими расчетами; $\lambda$ - коэффициент перевода размерности.

Отдельно нужно сказать об условии $\mathrm{H}=$ const, которое имеет место в течение суток. На высоконапорных станциях и в каскаде изменение нижнего бьефа, а значит, напора, составляет примерно $1 \%$, т. е. в этом случае погрешность пересчета колебаний напора приблизительно $1 \%$ и этим можно пренебречь. На каскадах нижний бьеф одной станции является верхним бьефом другой. Как известно, верхний бьеф меняется в меньших пределах при пропускании 1 м $3 /$ в воды из верхнего бьефа в нижний, так как зеркало верхнего бьефа гораздо больше, чем нижний бьеф.

На средне- и низконапорных станциях колебания напора более существенны, чем на высоконапорных станциях [15].

Однако в течение суток напор на любых ГЭС изменяется незначительно. Поэтому при выводе условий оптимизации чаще всего изменение напора в течение суток (при оперативном управлении) не учитывают.

Предлагаемый подход основывается, прежде всего, на корректном представлении дифференциальных характеристик (3) и (4). Действительно, данные характеристики должны быть производными не от расходов энергоресурсов, а от затрат (издержек), связанных с их использованием:

$$
\begin{aligned}
& U_{B}=C_{B} B, \\
& U_{Q}=C_{Q} Q,
\end{aligned}
$$

где $C_{\mathrm{B}}$ и $C_{Q}-$ стоимость (цена) топлива на тепловых станциях и стоимость гидроресурса на ГЭС соответственно.

Тогда выражения (2) и (3) будут рассчитываться следующим образом:

$$
\begin{aligned}
& b^{*}=C_{B} \frac{d B}{d Q}, \\
& q^{*}=C_{Q} \frac{d Q}{d N} .
\end{aligned}
$$


Что касается цены на топливо на тепловых станциях $C_{\mathrm{B}}$, то принципиальных сложностей с ее определением нет [5]. Даже в случае с эквивалентной тепловой станцией ее можно рассчитать (с некоторыми допущениями) как средневзвешенную.

Относительно стоимости гидроресурса, связанной с режимными особенностями работы ГЭС в ЭЭС, можно сказать, что эта задача никогда не решалась. Именно этому вопросу и будет уделено основное внимание в дальнейшем изложении материала.

Несмотря на то что данная задача относится к краткосрочной оптимизации режимов работы станций в энергосистеме в силу ограниченности энергоресурсов на ГЭС, она не может быть решена в отрыве от оптимизации долгосрочного режима работы ЭЭС [8]. Здесь под краткосрочной оптимизацией понимается суточная оптимизация, а под долгосрочной - водно-энергетический режим работы гидростанции в течение года с детальным учетом сезонных режимов ГЭС.

Таким образом, условие оптимального распределения нагрузки в гидротепловой энергосистеме в новой постановке можно представить в другом виде:

$$
\begin{aligned}
& b^{*}=q^{*}=i d e m, \\
& H=\text { const } \text {, } \\
& P_{C}=N_{T Э C}+N_{\Gamma \ni C}, \\
& N_{T Э C \text { min }} \leq N_{T Э C} \leq N_{T Э C \text { max }}, \\
& N_{\Gamma Э C \text { min }} \leq N_{\Gamma Э C} \leq N_{\Gamma Э C \text { max }} .
\end{aligned}
$$

Очевидны принципиальные отличия нового условия оптимизации (13) от предыдущего (2). Остановимся на них более подробно.

Здесь $b^{*}$ и $q^{*}$ определяются по (11) и (12) и являются производными от затрат (издержек), связанных с использованием расходов энергоресурсов на ТЭС и ГЭС соответственно.

Также в условии отсутствует проверка требования равенства среднесуточного расхода воды на ГЭС заданному расходу, полученного из водно-энергетического расчета в годовом разрезе [10]. Это связано с тем, что при построении ХОП ГЭС $\left(q^{*}\right)$ учтена мощность, с которой будет работать ГЭС в данный период года, а значит, учтен расход и напор воды. Поэтому проверка требования равенства среднесуточного расхода воды на ГЭС заданному расходу является излишней и итеративный характер расчетов, о котором было сказано выше, исчезает, что служит главным преимуществом предложенного подхода.

Кроме того, отсутствует проверка вида $\lambda=$ const исходя из того, что дифференциальные характеристики затрат на использование энергоресурсов на ТЭС (топлива) и на ГЭС (воды) имеют одинаковую размерность в денежном выражении.

И наконец, появляется реальное знание, какова цена гидроресурса, которая используется для покрытия баланса мощности в энергосистеме.

Все это позволяет для оптимального распределения нагрузки в гидротепловой энергосистеме использовать более понятные и корректные критерии оптимизации. Кроме того, знание стоимости водных ресурсов, которые используются для выработки электроэнергии на гидростанции, само по себе ценно и информативно. Оно дает возможность повышения эффективности управления как на самой гидростанции, так и в составе водно-хозяйственного комплекса (ВХК) в целом.

$$
-647-
$$




\section{3. Реализация предложенной модели}

Для решения необходимо построить линии безразличия для гидростанции для каждого месяца внутри года, так как мощность ГЭС, как известно, зависит от гидрографа реки и является изменчивой, т. е. таких линий будет 12 штук

Опишем алгоритм решения в соответствии с приведенной постановкой.

Первое уравнениее цели представлено ниже:

1 .

$$
\frac{1}{b}=\frac{1}{q} \text { при } \mathrm{U}=\mathrm{const},
$$

где $\mathrm{U}$ - линия безразличия.

Для определения значений $\mathrm{b}$ и q, которые необходимо использовать при построении линии безразличия, воспользуемся результатами выполнения водно-энергетического расчета, а именно значением $\mathrm{N}_{\text {гар. }}$. При этом необходимо отметить, что значение гарантированной мощности соответствует расчетной обеспеченности. Гарантированная мощность рассчитана на маловодный год.

Далее для найденного значения гарантированной мощности определяется значение Q:

$$
N_{\text {ГAP }}^{A}\left(Э_{\text {мес }}\right) \rightarrow Q
$$

при $\mathrm{N}_{\text {ГАР }}=$ const.

Далее по характеристике относительного прироста расхода воды можно определить соответствующее значение относительного прироста расхода воды (q).

$$
\begin{gathered}
q=\frac{d Q_{\Gamma \ni C}}{d N_{\text {гар }}} . \\
Q_{\Gamma \ni С} \geq Q_{\text {гар }} .
\end{gathered}
$$

2. При этом балансовые уравнения будут иметь следующий вид:

$$
P_{C}^{A}=N_{\Gamma A P}^{A}+N_{T \ni C}^{A} .
$$

3. Уравнение связи. Также при расчетах необходимо учесть такое требование, как выполнение энергетического баланса энергосистемы.

$$
B=f\left(N_{T Э C}\right), Q_{\Gamma \ni C}=f\left(N_{\Gamma \ni C}, H_{\Gamma \ni C}\right) .
$$

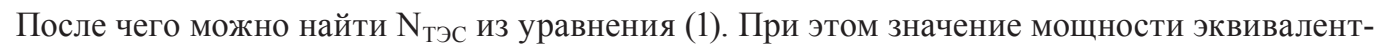
ной ТЭС получается как

$$
P_{C}^{A}-N_{\Gamma A P}^{A}=N_{T \ni C}^{A}
$$

Отсюда можно определить значение относительного прироста эквивалентной ТЭС. Для этого можно использовать характеристику относительного прироста расхода топлива эквивалентной ТЭС

$$
b=\frac{d B}{d N_{\text {ТЭС }}} .
$$

При построении кривых относительных приростов расхода воды на ГЭС и топлива на ТЭС текущие значения относительных приростов делили на средние значения относительного прироста расхода воды и топлива соответственно.

$$
-648-
$$


По этим значениям строим линию предельной полезности или безразличия (рис. 1).

Причем необходимо отметить, что для перехода от относительного прироста расхода воды к относительному приросту расхода топлива не используется коэффициент неопределенных множителей Лагранжа $\lambda$. Значение относительного прироста расхода воды на ГЭС q', лежащее на линии безразличия (рис. 1), будет равно соответствующему значению относительного прироста расхода топлива на ТЭС b', лежащего на той же линии, поскольку вода и топливо в данном случае будут обладать одной и той же ценностью для потребителя (выработка 1 Квт мощности). Это явное преимущество методики при решении оптимизационных задач для смешанных энергосистем, состоящих из гидравлических и тепловых, поскольку позволяет существенно сократить машинное время (требуется всего одно действие вместо пяти итераций) на решение не только поставленной задачи оперативного регулирования, но и оптимизационной задачи с целью обеспечения конкурентоспособности генерирующей компании на рынке.

При этом все соответствующие значения мощности для конкретного часа согласно суточному графику нагрузок лежат на данной линии безразличия в рамках рассматриваемого месяца [16].

Используя уравнение связи и зная значение $\mathrm{N}_{\text {гар }}$ для ГЭС, а также общую мощность энергосистемы для конкретного часа суток, определим мощность эквивалентной ТЭЦ, используя характеристику равенства относительных приростов расхода топлива (рис. 2 и 3).

4. При этом необходимо учесть уравнения ограничений:

$$
\begin{aligned}
& \mathrm{N}_{\text {TЭС min }} \leq \mathrm{N}_{\text {ТЭС }} \leq \mathrm{N}_{\text {ТЭС max }}, \\
& \mathrm{N}_{\text {ГЭС min }} \leq \mathrm{N}_{\text {ТЭС }} \leq \mathrm{N}_{\text {ГЭС max }} .
\end{aligned}
$$

5. Далее необходимо вывести оптимизационное уравнение $\mathrm{MR}=\mathrm{MC}$. Это есть не что иное, как критерий максимизации прибыли. При этом производитель будет максимизировать прибыль, производя продукцию в той точке, где предельный доход равен предельным издержкам. Этот руководящий принцип максимизации прибыли называется правилом равенства предельных издержек предельным доходам.

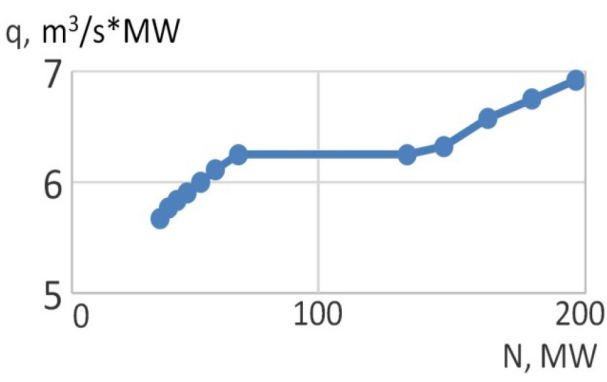

Рис. 2. Характеристика относительного прироста расхода воды НГЭС для работы ГЭС при напоре $17,9 \mathrm{M}$

Fig. 2. Incremental water rate characteristic for the Novosibirsk HPP with its operation under the head of $\mathrm{H}=17.9 \mathrm{~m}$

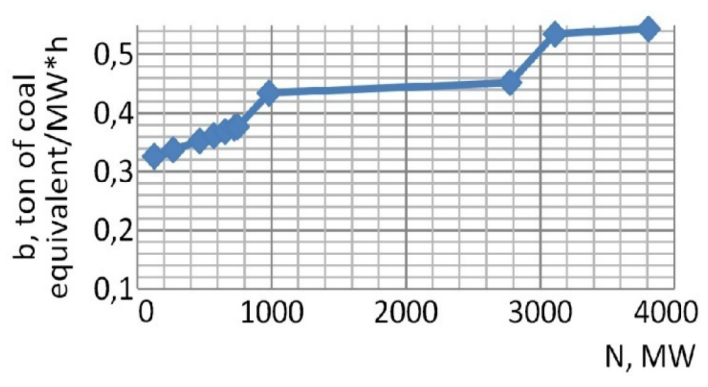

Рис. 3. Характеристика относительного прироста расхода топлива эквивалентной ТЭЦ для работы ГЭС при напоре 17,9 м

Fig. 3. Incremental fuel rate characteristic for the equivalent CHPP with HPP operation under the head o f $\mathrm{H}=17.9 \mathrm{~m}$. 
Для получения линии предельных издержек необходимо определить стоимость гидроресурса с учетом вынужденных режимов работы ГЭС на основе комплексного критерия экологоэкономической эффективности. Для этого необходимо выполнить такую последовательность действий.

Переходя к линии безразличия по предельным издержкам расхода воды и топлива, необходимо отметить, что угол наклона линии безразличия ( $\alpha)$ остается точно таким же, как и на рис. 1.

Отсюда можно определить стоимость гидроресурса для ГЭС, руководствуясь следующим графиком (рис. 4), на котором изображена линия безразличия для предельных издержек ГЭС и ТЭС.

При этом можно определить стоимость гидроресурса для ГЭС по следующему выражению:

$$
U_{q}=u^{*} q, \operatorname{tg} \alpha=\frac{1 / q}{1 / b}, u_{\text {водь }}=\operatorname{tg} \alpha * \frac{U_{B}}{u_{T}}
$$

где $\mathrm{U}_{\mathrm{q}}$ - предельные издержки на ГЭС; $\mathrm{U}_{\mathrm{b}}$ - предельные издержки на ТЭЦ; b - относительный прирост расхода топлива на ТЭЦ; q - относительный прирост расхода воды на ГЭС; Цводы - цена воды для ГЭС; цт - цена топлива для эквивалентной ТЭС.

На примере периода естественной приточности определим цену воды на ГЭС следующим образом: $u_{6}=\frac{U_{b}}{\operatorname{tg} \alpha^{*} q}=\frac{242,5}{0,072 * 6,05 * 3600}=0,1545 \frac{p y \sigma}{M^{3} / c e \kappa}$. Чтобы перевести цену воды в руб/ MBт*ч, необходимо определить предельные издержки на ГЭС для выдачи гарантированной мощности, т. е. составить пропорцию $U_{q}=U_{b} / \operatorname{tg} \alpha=242,5 / 0,072=3364,97$ руб/МВт*ч $=3,35$ руб/ кВт*ч. Такова стоимость гидроресурса с учетом технологических особенностей работы ГЭС.

Сведем все расчеты по стоимости гидроресурса для каждого месяца в течение года в одну общую табл. 1.

Практическая апробация предложенного метода расчетов на Новосибирской гидроэлектростанции помогла получить следующую стоимость гидроресурса для каждого месяца по се-

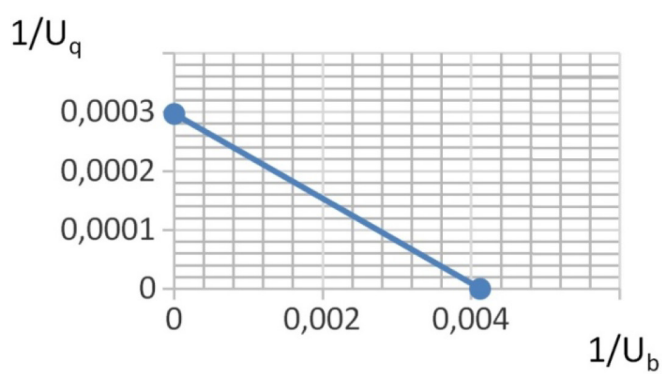

Рис. 4. Линия безразличия для периода бытового стока для предельных издержек (напор 17,9 м)

Fig. 4. The indifference line for marginal costs in the period of operation with natural river flow (the head is $17.9 \mathrm{~m})$ 
Таблица 1. Стоимость гидроресурса для каждого периода года с учетом режимных особенностей ГЭС

Table 1 . The cost of the hydro resource for each period of the year, with allowance the regime characteristics of the hydroelectric power station

\begin{tabular}{|l|l|}
\hline \multicolumn{1}{|c|}{ Период } & \multicolumn{1}{c|}{ Сработка } \\
\hline Месяц & Стоимость гидроресурса, Цводы (сработка) $=15,83$ коп $/ \mathrm{m}^{3} / \mathrm{c}$, \\
\hline 1 & 15,9642 \\
\hline 2 & 15,9159 \\
\hline 3 & 15,8357 \\
\hline 4 & 15,8457 \\
\hline Период & Наполнение \\
\hline Месяц & Стоимость гидроресурса, Цводы (межень) $=9,63$ коп $/ \mathrm{M}^{3} / \mathrm{c}$, \\
\hline 5 & 9,5345 \\
\hline 6 & 9,5555 \\
\hline 7 & 9,5555 \\
\hline 8 & 9,8765 \\
\hline Период & Естественная приточность \\
\hline Месяц & Стоимость гидроресурса, Цводы (ест.приточность) \\
\hline 9 & 11,3843 \\
\hline 10 & 11,2433 \\
\hline 11 & 12,4383 \\
\hline 12 & 12,1 \\
\hline
\end{tabular}

зонам года, а именно для периодов «Сработка», «Наполнение» и «Естественная приточность», по результатам расчетов, приведенных в табл. 1, средняя стоимость в период сработки -, средняя стоимость в период межени -, средняя стоимость в период естественной приточности -.

Так, стоимость воды на НГЭС для периода сработки 0,1583 руб/м³/c. Если перевести ее в часы, то получим $0,1583 * 3600=569$ руб/м³ , что сопоставимо с ценой 1 т бурого угля, равной 690-980 руб. за тонну в зависимости от сезона года.

Рассчитанные значения позволяют сравнить их со стоимостью энергетического топлива для ТЭЦ. Например, для бурого угля она равна 680-970 руб. за тонну, а кузнецкого угля порядка 1150 руб. за тонну. Рассчитанные значения стоимости воды на НТЭС меньше стоимости используемого энергетического топлива на ТЭС, что говорит об эффективности применяемой методики расчета. Это позволит в определенный период загрузить более экономичную гидроэлектростанцию вместо альтернативной теплоэлектростанции. Если рассчитать аналогичные показатели для кузнецкого угля, используемого на ТЭЦ, то получим, что для периода сработки на НГЭС-0,1583*3600 = 569 руб/м³, что в два раза меньше стоимости 1 т кузнецкого угля, равной 1200 руб. за тонну. Это свидетельствует об эффективности разработанной методики, поскольку позволит грузить более экономичную и экологичную ГЭС взамен тепловых электростанций.

Тогда предельные издержки для ГЭС определяться как 


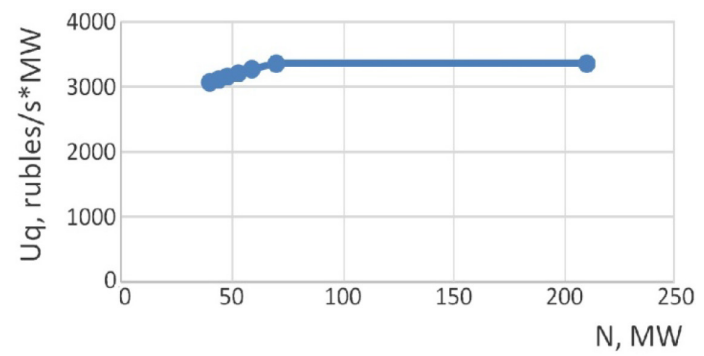

Рис. 5. Характеристики предельных издержек НГЭС для работы ГЭС при напоре 17,9 м

Fig. 5. Marginal costs characteristic for the Novosibirsk HPP with its operation under the head of $\mathrm{H}=17.9$ m. der the head of $\mathrm{H}=17.9 \mathrm{~m}$.

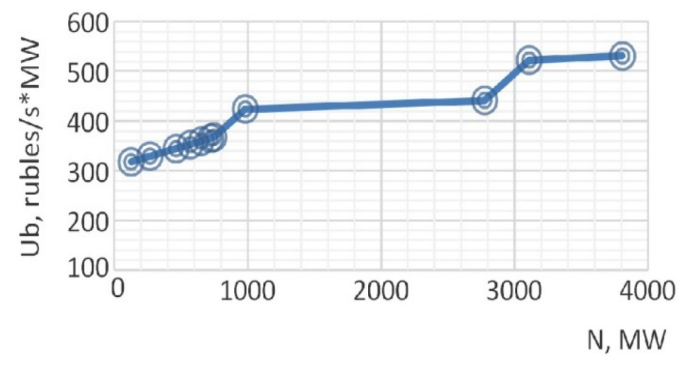

Рис. 6. Характеристики предельных издержек эквивалентной ТЭЦ для работы ГЭС при напоре $17,9 \mathrm{M}$

Fig. 6. Marginal costs characteristic for the equivalent CHPP with HPP operation under the head of $\mathrm{H}=17.9 \mathrm{~m}$

$$
M C=u_{\text {воды }} * q .
$$

Для примера на рис. 5 и 6 приведены предельные издержки для НГЭС и эквивалентной ТЭС.

Совместно решая систему уравнений, описывающих кривые предельных издержек и предельных доходов, можно определить оптимальные значения среднемесячной выработки (Э для каждого сезона и среднесуточные мощности:

$$
N_{\text {onm }}=Э_{\text {onm }} / t_{\text {мec }}
$$

где $\mathrm{t}_{\text {мес }}-$ среднее число часов в месяц (720 ч).

Для определения оптимальных режимов загрузки НГЭС для каждого конкретного периода года использовался критерий максимизации прибыли (табл. 2).

Практическая апробация разработанной методики на НГЭС позволила получить следующие цены воды для ГЭС по сезонам года (паводок, межень и работа на бытовом стоке): цена воды для НГЭС в период паводка составляет 0,1124 руб/м³ $/ \mathrm{c}$, для межени $-0,1583 \mathrm{py} / \mathrm{m}^{3} / \mathrm{c}$, для работы на бытовом стоке - 0,1545 pyб/м³/c.

Более того, необходимо отметить, что стоимость воды на Новосибирской ГЭС сопоставима со стоимостью топлива на ТЭЦ, что свидетельствует о правильности проведенных расчетов.

Таблица 2. Оптимальные значения мощностей по сезонам года для НГЭС

Table 2. Optimal values of power output for the Novosibirsk HPP by year seasons

\begin{tabular}{|l|c|c|c|}
\hline \multicolumn{1}{|c|}{ Сезон года } & $\begin{array}{c}\text { Режим работы ГЭС } \\
\text { при напоре } \mathrm{H=14,05} \mathrm{м}\end{array}$ & $\begin{array}{c}\text { Режим работы ГЭС } \\
\text { при напоре } \mathrm{H=17,5} \text { м }\end{array}$ & $\begin{array}{c}\text { Режим работы ГЭС } \\
\text { при напоре } \mathrm{H}=17,9 \text { м }\end{array}$ \\
\hline Норма прибыли & $0 \%$ & $0 \%$ & $0 \%$ \\
\hline Мощность, МВт & 305 & 70 & 124 \\
\hline Электроэнергия, МВт*ч & 219600 & 50400 & 3700 \\
\hline $\begin{array}{l}\text { Заявленная цена, руб/ } \\
\text { МВт*ч }\end{array}$ & 3600 & 3786 & 660672000 \\
\hline Доход, руб. & 2371680000 & 1335700800 & \\
\hline
\end{tabular}


Результаты расчета стоимости гидроресурса для разных сезонов оказались того же порядка, что и топливо на теплоэлектростанциях. На основе полученных цен на воду была получена кривая предельных издержек на НГЭС. После была проведена оптимизация НГЭС для каждого сезона на основе критерия максимизации прибыли, в результате которой оптимальная выработка мощности для НГЭС составляет для режима работы ГЭС при напоре $\mathrm{H=14,05}$ м 305 MBT, а цена продаж в этом случае будет равна 3600 , при напоре H=17,5 м - 70 MBT, а цена продаж - 3786, при напоре $\mathrm{H=17,9}$ м - 124 МВТ с ценой продаж 3700.

При сравнении с традиционным методом неопределенных множителей Лагранжа, используемого для оптимизации в смешанных энергосистемах, можно отметить, что в разработанном методе существенно сокращается количество итераций и машинное время на решение поставленной задачи (одна итерация против пяти в методе неопределенных множителей Лагранжа) [16].

Далее проводилась проверка правильности разработанной методики путем сравнения полученных результатов по наивыгоднейшему распределению нагрузки смешанной энергосистемы между эквивалентной ТЭС и ГЭС по предложенной авторами методике и традиционно используемому методу неопределенных множителей Лагранжа.

Согласно традиционной методике неопределенных множителей Лагранжа получаем для периода наполнения следующее распределение нагрузки энергосистемы между эквивалентной ТЭЦ и НГЭС для периода наполнения (рис. 7).

Причем необходимо отметить, что погрешность отклонения среднесуточного расхода

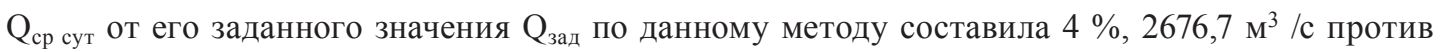
$2767 \mathrm{~m}^{3}$ /с для периода наполнения.

Для разработанной авторами методики можно привести следующие результаты (рис. 8).

Погрешность отклонения $\mathrm{Q}_{\text {ср сут }}$ от $\mathrm{Q}_{\text {зад }}$ по данному методу составила $9 \%, 3033 \mathrm{~m}^{3} / \mathrm{c}$ против $2767 \mathrm{~m}^{3} / \mathrm{c}$ для периода наполнения. При этом возросла доля НГЭС в покрытии суточного графика нагрузки на $12 \%$.

Таким образом, результаты, полученные по разработанной методике, находятся внутри диапазона согласно традиционному методу распределения нагрузки смешанной энергосисте-

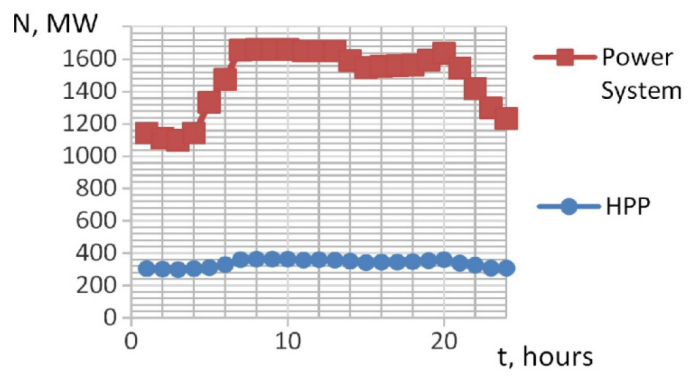

Рис. 7. Распределение нагрузки в смешанной энергосистеме по методу неопределенных множителей Лагранжа для периода наполнения

Fig. 7. Schedule of daily power distribution of the SGC between the Novosibirsk HPP and the equivalent CHPP for the high-water period according to the Lagrangian multiplier method

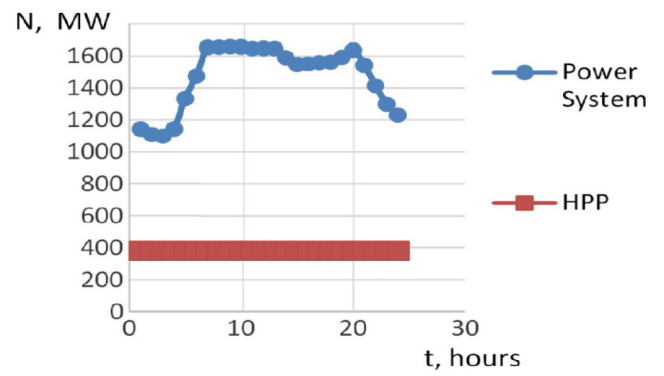

Рис. 8. Распределение нагрузки в смешанной энергосистеме по методике предельной полезности для периода наполнения

Fig. 8. Schedule of daily power distribution of the SGC between the Novosibirsk HPP and the equivalent CHPP for the high-water period 
мы, т. е. методу неопределенных множителей Лагранжа [9]. Это свидетельствует о достоверности полученных результатов и правильности разработанного подхода.

\section{Заключение}

По результатам проведенного научного исследования можно отметить следующее:

- проанализирована традиционная методика наивыгоднейшего распределения нагрузки в гидротепловой энергосистеме по методу неопределенных множителей Лагранжа и выявлены ее недостатки, связанные с множественностью итераций, а также с отсутствием реальной стоимости гидроресурса;

- предлагаемый подход основывается, прежде всего, на корректном представлении дифференциальных характеристик ГЭС и эквивалентной ТЭЦ. Действительно, данные характеристики должны быть производными не от расходов энергоресурсов, а от затрат (издержек), связанных с их использованием. Это явное преимущество методики при решении оптимизационных задач для гидротепловых энергосистем, состоящих из ГЭС и эквивалентной ТЭЦ, поскольку позволяет в полной мере учесть режимные особенности ГЭС и существенно сократить машинное время (требуется всего одно действие вместо нескольких итераций) на решение не только поставленной задачи оперативного регулирования, но и оптимизационной задачи с целью обеспечения конкурентоспособности генерирующей компании на рынке;

- обосновано использование теории предельной полезности для определения стоимости гидроресурса с учетом режимных особенностей работы ГЭС для оптимального покрытия баланса ЭЭС, что ранее никогда не исследовали;

- осуществлена расчетная и экспериментальная проверка разработанных подходов и методов, а также проведена реализация основных положений исследования на конкретных объектах, по результатам которых было выявлено увеличение доли ГЭС в покрытии суточного графика нагрузки на $12 \%$.

\section{Список литературы / References}

[1] Sekretarev U. A., Mitrofanov S. V. Decision support system for hydro unit commitment, 2nd Int. Conf. On Ind. Eng. Applications and Manufacturing. Chelyabinsk: IEEE, 2016, 7911424.

[2] Sekretarev Y. A., Panova Y. V. Investigations of possible using a generalized fuzzy interval for analyzing operating conditions of power equipment at hydropower plants, 2nd Int. Conf. On Ind. Eng. Applications and Manufacturing. Chelyabinsk: IEEE, 2016, 7911025.

[3] Sekretarev Y.A., Panova Y.V. Development of the intelligent decision support system for situation management of hydro units, 14th Int. Sci.-Tech. Conf. on Actual Problems of Electronic Instrument Eng. APEIE. Novosibirsk: IEEE, 2018, 384-8.

[4] Sultonov S., Sekretarev Y., Mitrofanov S. Implementation of the method of lagrange for optimal modes of energy system of Tajikistan, Applied Mechanics and Materials, 2015, Vol. 698: Electrical Engineering, Energy, Mechanical Engineering, EEM 2014, 726-731, DOI: 10.4028/www. scientific.net/AMM.698.726.

[5] Moshkin B.N., Myatezh T.V., Sekretarev Y.A., Averbukh M.A. Mathematical model of managing of the generating company on the criterion of the profit maximization, IOP Conf.. Ser.: Mater. Sci. Eng, 2019, 552012016. 
[6] Sekretarev Y., Sultonov S. and Nazorov M. Optimization of long-term modes of hydropower plants of the energy system of Tajikistan, 2nd Int. Conf. on Industrial Eng Applications and Manufacturing. Chelyabinsk: IEEE), 2016, 7911428.

[7] Ghamgeen I. R. Inflows and energy demands of dokan hydropower reservoir operation under stochastic conditions, 13th Ieee Conf. on Ind. Electr. and Appl. Wuhan: IEEE, 2018, 8397804.

[8] Seif Azghandi, Kenneth Mark Hopkinson, Kennard Robert Laviers. Benchmarking approach for empirical comparison of pricing models in DRMS, The Journal of Engineering, 2016, 8 p.

[9] Marc A. Rosen, Dr Seama Koohi-Fayegh. Cogeneration and District Energy Systems: Modelling, Analysis and Optimization, 2016. 344 p.

[10] Lukutin B. V., Shandarova E. B., Matukhin D.L., Makarova A.F. and Fuks I. L. Operation modes of a hydro-generator as a part of the inverter micro hydropower plant, IOP Conf. Ser.: Mater. Sci. Eng, 2016, 124(1), 012070.

[11] Zhang L., Wu Q., Ma Z., Wang X. Transient vibration analysis of unit-plant structure for hydropower station in sudden load increasing process, Mech. Syst. and Signal Processing. 2019, 120, 486-504.

[12] Chang J., Wang X., Li Y., Wang Y., Zhang H. Hydropower plant operation rules optimization response to climate change Energy, 2018. 160886-97.

[13] Balzannikov M.I. Research on flow in water intake of a run-of-river hydropower plant, MATEC Web Conf, 2017, 11700013.

[14] Секретарев Ю.А., Мятеж (Чекалина) Т. В., Мошкин Б. Н. Оптимизация режимов работы генерирующей компании на базе ТЭЦ по выработке электроэнергии на основе критерия максимизации прибыли, Известия высших учебных заведений. Электромеханика, 2016, 4(546), 82-88 [Sekretarev U.A., Myatezh T. V., Moshkin B. N. Administration functioning power generation companies based on thermal electrical power station on maximization profit criterion. Proceedings of higher educational institutions. Electromechanics, 2016, 4(546), 82-88 (in Russian)]

[15] Sekretarev Y., Sultonov S., Shalnev V. Optimal control mode of the Vakhsh hydropower reservoirs to reduce electricity shortage in Tajikistan, Appl. Mech. and Materials, 2015, 792 446-50.

[16] Sekretarev U. A., Myatezh T. V., Gorshin A.V. Justification of the water price at hydropower plants based on its operational features according to the maximization profit criteria [Electronic resource] - Access: https://ieeexplore.ieee.org/document/9271220/authors\#authors. Title from screen - DOI: 10.1109/FarEastCon50210.2020.9271220. International multi-conference on industrial engineering and modern technologies (FarEastCon): [proc.., Vladivostok: IEEE, 6-9 Oct. 2020, 6 p. 